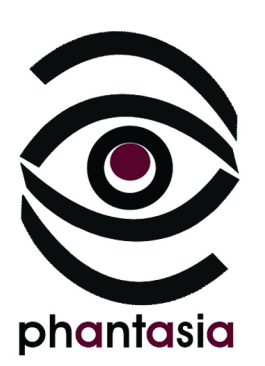

\title{
Image ou événement? Quelques destins français de la psychanalyse
}

\author{
Claire Pagès \\ Laboration ICD, Université de Tours
}

Nous souhaitons ici développer une réflexion touchant l'ambivalence de la pensée psychanalytique de l'événement, telle du moins qu'on peut la thématiser à partir des textes de Freud, en présentant les oscillations qui sont les siennes non pas comme un motif d'invalidation mais comme une ressource pour une pensée soucieuse de faire doit et place aujourd'hui à l'événement.

Cette difficulté d'interprétation de la théorie freudienne est régulièrement le cœur de discussions qui excèdent le champ psychanalytique. Par exemple, dans sa récente discussion avec l'interprétation freudienne du rêve, publiée sous le titre L'interprétation sociologique des rêves (2018), le sociologue Bernard Lahire promeut une théorie dispositionnaliste-contextualiste du rêve qui rapporte son contenu à la fois à un passé intériorisé sous la forme de traces mnésiques mais aussi aux nouveaux contextes dans lesquels s'inscrit l'individu. Si bien des éléments de la théorie freudienne du rêve restent précieux, d'autres ne laissent pas d'en déformer la véritable portée. Parmi eux, "l'événementialisme» de cette théorie, soit la tendance à privilégier le poids de l'événement et à rattacher le rêve comme les autres logiques mentales et comportementales à un événement marquant ou à une personne marquantes du passé. B. Lahire tend à montrer, au contraire, que le rêve prend davantage racine dans « des séries relativement cohérentes d'événements ou de séquences d'événements, ou des types relativement analogues de personnes ${ }^{1}$ qui participent à la constitution dans la durée de dispositions individuelles.

Il ne s'agit pas de discuter ici cette analyse qui possède bien d'autres représentants. Mais on peut déjà contraster cette position avec le reproche inverse, adressé parfois à la psychanalyse, de méconnaître l'événementialité. Les choses sont en réalité plus compliquées : la théorie psychanalytique, si cela a un sens de parler de façon aussi générale, est tantôt interprétée comme ouverture à l'événement, c'est-à-dire appréhendant la vie psychique du sujet comme tributaire d'une expérience, de ce qui lui arrive dans le monde, ce déterminant d'extériorité pouvant relever de l'accident, du contexte, de la rencontre, etc., tantôt comme pensée du fantasme, logique des images sans origine, soit d'une interprétation de la vie psychique du sujet qui indexe celle-ci à des structures ou à des logiques internes autonomes indépendantes de la réalité. L'exigence de penser l'événement qui s'impose à nombre de philosophes du second XXe siècle engage une attitude ambivalente à l'égard de la psychanalyse et cela parfois chez un même auteur. Tantôt la prise en compte de l'événement exige de rompre avec la psychanalyse, tantôt elle impose de s'inspirer de ses modèles et de ses concepts.

L'étude de certains destins français de la psychanalyse dans le courant du second XXe siècle nous semble permettre de revenir sur ce désir d'événement qui a marqué la pensée, en particulier philosophique, pendant cette période, mais aussi de préciser une ambivalence - image ou événement : la vie psychique se comprendelle comme développement de fantasmes ou comme intériorisation d'une expérience du réel ? - qui pourrait ne pas être seulement celle de la réception : n'y a-t-il pas au cœur de la théorie psychanalytique, telle que Freud a commencé à la mettre en œuvre, un discours clivé qui indexe le cours de la vie psychique tantôt à

${ }^{1}$ LAHIRE Bernard, L'Interprétation sociologique des rêves, 2018, Paris, La Découverte, p.182. 
des événements tantôt à des fantasmes? Thématiquement, cette discussion nous semble pouvoir enrichir une réflexion générale sur l'image et l'imagination, dans la mesure où la notion d'événement, dans le cadre de ce débat, est d'abord envisagée comme alternative à la logique fantasmatique comprise comme logique autonome des images inconscientes. Nous montrerons progressivement que ce dualisme peut être remis en question.

Il nous faut ici ajouter une première remarque touchant les concepts centraux de la discussion : la théorie psychanalytique freudienne et d'inspiration freudienne, plus qu'un concept d'événement unifié dont elle ne dispose pas et dont elle n'use pas, s'est efforcée de concevoir à partir de la pratique, à partir du champ de la clinique, des figures de l'événementialité qui ne font pas système mais dont nous interrogerons les différences. La théorie ou les théories de l'événement - nous prenons la responsabilité de ce terme qui n'est pas chez Freud un concept mais qui est employé pour désigner une impression ou expérience vécues servent à rendre intelligible un réel clinique - un symptôme, une pathologie, une souffrance -, à comprendre son mécanisme pour entreprendre de le soigner. Il n'y a donc pas nécessité d'unifier le propos en synthétisant tous les régimes d'événement et en les subsumant sous un concept unique, Freud définissant le travail du psychanalyste comme un exercice d'analyse - travail chimique d'analyse de l'activité psychique en ses composants élémentaires - qui ne participe d'aucune synthèse ultérieure, d'où son opposition à toute forme de psycho-synthèse ${ }^{2}$.

Notre seconde remarque d'ordre épistémologique est la suivante: Freud ne constitue pas non plus la notion d'image en concept, ce que fera par contre Lacan, en particulier à partir du texte de la conférence de 1949 sur le stade du miroir. Néanmoins, ses écrits présentent divers développements permettant d'enrichir une réflexion sur l'image. Une première option pourrait consister à suivre la distinction établie par Freud entre mot et image, entre représentation de mot et représentation de chose, qui fait le partage entre d'une part les systèmes préconscient et conscient et, d'autre part, le système inconscient : si l'inconscient est pour Freud pensée en images, et le rêve de façon élective compris comme irruption d'images - le franchissement du seul de la conscience va de pair avec l'adjonction d'une représentation de mot à l'image. Se rattache à cela la question freudienne de la figuration, soit de ce qui permet d'exister dans l'inconscient comme image (par exemple la formation d'un investissement). Mais nous avons choisi de suivre une autre piste : une deuxième pensée de l'image se trouve développée en creux par Freud et opposée à tout ce qui fait événement. Ici, une trace mnésique déjà présente génère un état d'affect, par exemple de l'angoisse, et s'oppose à la logique causale pour laquelle un état psycho-affectif est engendré par un événement ${ }^{3}$. De ce fait, l'image pend la forme du fantasme qui désigne ce qui « surgi de manière apparemment spontané » ${ }^{4}$ et qui s'oppose à ce qui est étayé sur des objets réels ou qui résulte d'un acte. Ainsi, les hystériques qui souffrent de réminiscences fantasment le plus souvent les scènes de séduction plutôt quelles ne les tirent de leur expérience vécue 5 .

Nous proposons dans un premier temps d'exposer pourquoi la théorie psychanalytique, freudienne en particulier, a pu être jugée rétive à l'accueil de l'événement, en raison justement du poids qu'elle accorde au fantasme. Nous verrons ensuite que d'autres traits de la théorie psychanalytique ont inspiré des penseurs français soucieux de l'événementialité. Nous proposerons d'interpréter cette incertitude touchant l'usage qu'on peut faire de la psychanalyse pour penser l'événement, avant de suggérer quelles pourraient être, selon l'hypothèse qui est la nôtre, les principales contributions conceptuelles de la théorie psychanalytique freudienne à une pensée contemporaine de l'événement.

(1) La pensée freudienne des névroses traumatiques a certes constitué une source d'inspiration pour les penseurs de l'événement. Les névroses traumatiques nous exposent en effet à un cas limite, exceptionnel, dans la théorie des névroses, car elles se développent d'abord, à la suite d'un effroi ou d'un accident grave,

\footnotetext{
${ }^{2}$ Voir FreUd, « Les voies de la thérapie psychanalytique », 1919, p. 97-108, Euvres Complètes XV, Paris, PUF, 1996, p. 102. Et « De la psychogenèse d'un cas d'homosexualité féminine », 1920, p. 233-262, OC XV, op. cit., p. 258.

${ }^{3}$ Voir FreUD, «Inhibition, symptôme et angoisse », 1926, p. 203-286, Euvres Complètes, Psychanalyse, volume XVII, Paris, PUF, 1992, p. 211.

${ }^{4}$ FREUD, La psychopathologie de la vie quotidienne, Paris, NRF Gallimard, Connaissance de 1'inconscient, 1997, p. 419-420.

${ }^{5}$ Il ne s'agit pas pour Freud de nier l'existence de névroses traumatiques mais le périmètre de celles-ci se trouve néanmoins très circonscrit. Voir PAGÈS Claire, «Penser les conjonctures et les contextes et inventer des dispositifs », p. 481-493, Dossier « La psychanalyse dans la culture », Revue Française de psychanalyse, n`81, 2017/2.
} 
« sans la moindre relation avec un conflit du moi ${ }^{6}$ et elles semblent confronter le psychisme à un danger qui le menace de l'extérieur'. Il parait alors difficile de l'analyser avec les outils classiques - refoulement, mécanismes de défense, formations de compromis, etc. - et, quoi qu'en dise Freud parfois, l'unification de la théorie des névroses fait alors problème ${ }^{8}$.

Pourtant, dans l'ensemble, le modèle métapsychologique de développement défini par Freud a été compris comme un modèle monologique ${ }^{9}$, monadique ${ }^{10}$ ou monadologique ${ }^{11}$. Norbert Elias parle de l'homme freudien comme d'un "homo clausus ${ }^{12}$ pourvu de tendances et de désirs endogènes. Se trouve ainsi diversement désigné le fait que Freud aurait envisagé l'être humain comme une unité fermée sur ellemême, ne percevant pas que le développement de ses différentes fonctions, telle la maîtrise de soi, sont tributaires tout au long de l'existence des processus collectifs dans lesquels l'individu se trouve inscrit. La société semble alors pour le sujet une simple «toile de fond» selon l'expression d'Elias. Solidairement, la logique des fonctions psychiques se trouverait appréhendée sur le modèle des fonctions de l'organisme, l'inconscient, le ça conçus comme des entités "sans histoire », de ce fait indépendantes de la destinée relationnelle de l'individu, comme si ce dernier était animé de pulsions et d'instincts «à l'état brut » et non pas socialisées. À ce modèle, on impute donc une certaine ignorance de l'événementialité.

Un redéploiement des concepts et des pratiques psychanalytiques semble alors s'imposer à mesure que se fait en particulier plus criante l'irruption de l'économico-politico-social sur la scène de la santé mentale. René Kaës ${ }^{13}$ a ainsi par exemple initié tout un travail sur les violences d'État (ainsi que sur le génocide arménien) qui témoigne de sa prise en compte des limites d'un modèle métapsychologique relativement internaliste pour penser l'événement politique traumatisant. Il s'est interrogé sur la "catastrophe sociale » qu'ont représenté en Argentine les années de dictature entre 1976 et 1983. Cette catastrophe met la psychanalyse en crise: si fait défaut une pensée des rapports de l'histoire, du politique et du psychanalytique, on est confronté à l'impossibilité épistémique de comprendre du point de vue de la psychanalyse l'incidence de ces violences dans la psyché. Penser les irruptions et effractions issues de la violence de l'histoire, du politique ou de l'ordre économique exige ainsi de reconnaître la réalité d'une étiologie sociale, véritable "déterminant d'extériorité » dans le trouble psychique : de reconnaître la nature et l'origine de ces violences «hors du champ intrapsychique ${ }^{14}$. Ce développement rejoint le souci de l'incidence réelle, profonde et spécifique du contexte sociopolitique. En effet, nombre de critiques et d'analystes français de la psychanalyse freudienne associent une mise en avant du caractère objectivement traumatisant de l'environnement - et non imaginairement, fantasmatiquement ou symboliquement - à la mise en cause chez Freud d'une minoration de l'accidentalité pure et simple.

Dans Les Nouveaux blessés, Catherine Malabou vient reposer ce problème car il lui importe de comprendre le phénomène de désertion observable d'abord chez des malades cérébraux mais aussi chez d'autres types de traumatisés (le spectre des affections neurologiques évoqué dans le livre est large, allant de la maladie d'Alzheimer à celle de Parkinson, des blessés neurologiques aux traumatisés de guerre, etc.). Se posait la question de l'étiologie des souffrances de ces nouveaux blessés. Ce sont en réalité, dans bien des cas, d'anciens blessés dont la souffrance spécifique et l'étiologie neurologique sont restées longtemps ignorées. Or, ces blessés constitueraient ce que la psychanalyse freudienne ne peut entendre ou comprendre. Il s'agit de confronter la psychanalyse à un impensable pour elle, à son impuissance constitutive à penser par

\footnotetext{
${ }^{6}$ FREUD, «Introduction à Sur la psychanalyse des névroses de guerre », 1919, p. 217-223, OC XV, op. cit., p. 221.

${ }^{7}$ Ibid., p. 223.
}

${ }^{8}$ Néanmoins, le symptôme réactionnel constitue encore, selon certains lecteurs, une manière pour Freud de penser un événement traumatique en première personne, soit un traumatisme que la psyché fait sien. Voir BERNET Rudolf, «Le sujet traumatisé », p. 269-293, Conscience et existence, Perspectives phénoménologiques, Paris, PUF, Épiméthée, 2004, p. 290.

${ }^{9}$ Honneth Axel, La Lutte pour la reconnaissance, Paris, Éditions du Cerf, Passages, 2007, p. 119.

${ }^{10}$ Benjamin Jessica, Les Liens de l'amour, Paris, Métailié, 1992, p. 38.

${ }^{11}$ Adorno Teodor. W., La Psychanalyse revisitée, Paris, Éditions de l'Olivier, Penser/rêver, 2007, p. 39.

${ }^{12}$ Elias Norbert \& SCOTSON John L., Logiques de l'exclusion, Paris, Fayard, 1997, p. 59.

${ }^{13}$ Psychanalyste et professeur émérite de psychologie et psychopathologie cliniques à l'Université Lyon II, il s'est orienté vers la psychologie sociale des groupes (approche clinique des groupes restreints) puis vers la psychanalyse groupale.

${ }^{14}$ KAËS René, Violence d'État et psychanalyse, Paris, Dunod, 1989, p. XII-XIII. Voir Claire Pagès, « Aux croisements du psychique et du social : un usage théorique de la psychodynamique du travail», p. 151-168, Revue Travailler, Section « Théorie », $\mathrm{n}^{\circ} 31,2014$. 
exemple l'état de stress post-traumatique et plus largement l'événementialité cérébrale (qui affecte le cerveau sans faire signe vers une vérité du sujet).

Pourquoi la psychanalyse s'est-elle construit un impensable et qu'est-ce qui précisément lui échappe ? Catherine Malabou propose de distinguer les événements sexuels et les événements qui, portant atteinte aux affects cérébraux, sont irréductibles aux premiers. En effet, l'événement chez Freud serait défini par un mélange d'exogène et d'endogène, la sexualité en constituant le paradigme et le fond général. Cette définition se double d'une priorité causale accordée à l'endogène: ainsi la névrose est-elle présentée systématiquement comme la réactivation d'un conflit affectif, soit comme l'activation d'un mécanisme de défense. Cela se traduit également dans la distinction faite par Freud entre causes déterminantes et facteurs déclenchants, les événements extérieurs étant cantonnés à jouer ce second rôle : «c'est toujours la sexualité, comprise comme causalité et régime événementiel spécifiques, qui triomphe finalement de l'accident brut, de l'effraction pure... ${ }^{15}$. Or, l'événement cérébral possède principalement une dimension d'extériorité à l'égard du psychisme agressé et une extériorité bien plus consistante. Non seulement ces accidents psychiques ne sont pas transposables dans le langage de la sexualité, mais ils sont également impensables du point de vue du primat de l'intériorité. Ce que Catherine Malabou nomme le régime de la cérébralité définit donc un type d'événement singulier : « la cérébralité n'autorise toutefois pas l'assignation interprétative de la lésion ou du trauma à un "conflit antérieur". Elle n'admet pas non plus la précellence accordée à l' "ennemi intérieur". La cérébralité détermine le régime de l'événement désastreux qui ne joue aucun rôle dans un conflit affectif censé le précéder ${ }^{16}$. La théorie freudienne est donc empêchée de comprendre ce qui se joue dans la blessure cérébrale, mais aussi, dans un tout autre registre, dans de nombreux traumatismes possédant des causes sociopolitiques, si bien Freud ne serait pas au fond un penseur de l'accident. Si ces deux formes de traumatismes sont sans rapport, elles ont pourtant en commun de confronter le sujet à une agression extérieure, qui ne constitue pas un avatar de la logique de son désir ou une version de ses fantasmes.

Le type d'événement caractéristique de la cérébralité est marqué par l'effacement du caractère intentionnel du traumatisme. Plus précisément, il confronte la psyché à une absence de sens des événements qui sont pourtant les siens, car, soudain et aléatoire, il se soustrait à la prise du fantasme : "On ne fantasme pas une attaque cérébrale, on ne se la représente même pas. La cérébralité est ainsi la causalité de l'accident neutre et destructeur - sans raison ${ }^{17}$. Celle-ci possède des répercussions psychiques mais il n'y en a pas de mise en récit possible, car survient un changement « sans précédent », qui donne lieu à une métamorphose, à une nouvelle personne. Il n'y en a pas alors d'interprétation possible. C'est pourquoi « la cérébralité désigne le régime d'événementialité qui reconnaît le poids psychique de l'accident dénué de signification ${ }^{18}$.

La psychanalyse manquerait l'événementialité faute d'une pensée du "il arrive » indépendante de sa signification. Que ce soit dans «La scène de l'écriture ", La Carte postale ou le court texte d'Éperons " "J'ai oublié mon parapluie" ", Jacques Derrida a indiqué que, si la théorie psychanalytique échoue souvent à penser l'événement, c'est qu'elle appréhende et interprète tout événement psychique comme un message. En effet, pour Freud, tout semble faire sens dans la psyché : tout événement serait un message, dont le sens, inévident, est à déchiffrer. En somme, tout événement psychique se destinerait à une réception-transcription. En effet, cette affirmation du sens implique une cible ou une instance de lecture, du moins la possibilité toujours supposée de son intellection. Il n'y aurait pas de message, vain, indéchiffrable, perdu, inarchivable ou dépourvu de signification. À cela, Derrida a vigoureusement opposé la réalité de l'absence de destination et l'existence d'événements ou de messages qui se perdent et ne sont jamais traités comme signifiants. Dans les «Envois» de la Carte postale, il compare ainsi le traitement psychanalytique des significations au fonctionnement des postes ${ }^{19}$, comparant la psychanalyse à une poste centrale qui ne reconnaîtrait jamais ses ratés. Toutes deux affirmeraient qu'un message arrive toujours à destination ${ }^{20}$. Le dispositif psychanalytique rejoindrait, de ce fait, l'histoire hégélienne, tous deux servant de garanties indéfectibles au sens :

Ils ont beau le dénier, le «sens de l'histoire » n'est pas loin, quelques stations ou stases postales dans l'inconscient, quelques complications topiques supplémentaires et nous y sommes, nous n'en sommes

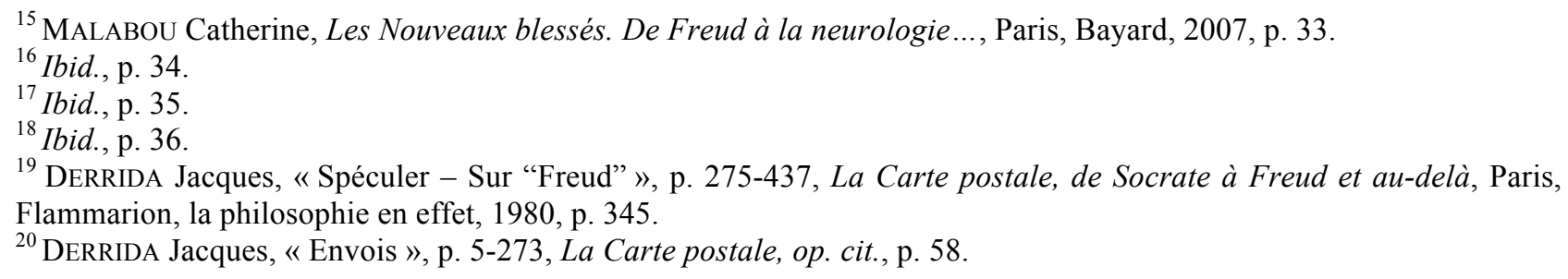


jamais sortis, de l'idéalisme spéculatif. Dès que cela arrive à destination, l'histoire aura eu un sens, et

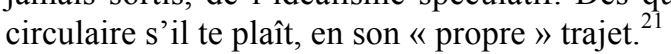

C'est alors comme par hasard - accidentellement - que la psychanalyse freudienne se rend alors sensible à l'événement. C'est ce que semble d'abord indiquer Jean-François Lyotard dans sa lecture de L'Homme aux rats, qui dénonce d'abord dans l'analyse de cas chez Freud la tendance à étouffer la voix affectuelle du patient au profit de l'articulation d'une lexis communicable, générale. Si la «phonè » et l'affect sont trahis, c'est que Freud les tient pour des référents dont il faut déchiffrer le sens. Est visée chez Freud comme source de son oubli une pensée représentative qui détermine intégralement le réel pour lui conférer un sens : « Avec le mot Rat, Freud, piqué au jeu du démêlage, pense avoir trouvé le nom de la navette qui court d'un fil à l'autre $»^{22}$. L'analyse du cas de L'homme aux rats permet néanmoins d'y dégager également un instant d'attention à l'événement de l'enfance. En dépit de la volonté freudienne d'écrire l'histoire d'un cas, de théoriser les affects d'Ernst, de fléchir sa voix pour la faire sonner clairement, Lyotard pointe un moment où Freud se rend à l'irréductibilité de cette voix, à son caractère inarticulable, inflexible, à son caractère anhistorique, neutre, inadressé ${ }^{23}$. L'enfance serait dans cette voix affectuelle d'Ernst, qui n'est signal que d'elle-même, qui n'a pas d'histoire, parle toute seule et ne peut être racontée faute de devenir affect mort ou en troisième personne comme la naissance. Contre la voix narrative et interprétante de Freud qui étouffe la voix affectuelle d'Ernst, est mis en avant le seul moment où l'analyste entend cette phonè : "Dans cette cacophonie, le miracle s'accomplit, la phônè se fait entendre et s'entend, l'affect s'adresse, la voix de Freud tinte à la voix d'Ernst... $»^{24}$. C'est pourtant comme malgré lui et malgré le dispositif théorique qui est le sien qu'un instant Freud entendrait cet événement qu'est la phonè.

En quoi consiste ici l'événement ? En un traumatisme d'abord, un accident grave. Il désigne l'irruption violente ou accompagnée d'effroi d'un dehors (choc, accident mais aussi irruption réelle du social sous la forme de la violence de l'histoire, du politique ou de l'ordre économique) dans un dedans. Cet événement fait effraction, il possède principalement une dimension d'extériorité mais n'a pas de caractère intentionnel ou se trouve dénué de signification. Qu'est-ce qui, selon ces penseurs français contemporains, dans la théorie psychanalytique, fait alors obstacle à la saisie d'un tel régime de l'événementialité et nécessite qu'on l'abandonne pour pouvoir y faire droit? Manifestement, le modèle métapsychologique freudien relativement internaliste, accordant priorité ou primat à l'intériorité. L'événement psychique (sexuel) ne saurait être, d'une part, qu'un mélange d'exogène et d'endogène, et, de surcroit, il se définirait comme un message à décrypter.

(2) Néanmoins, la psychanalyse apparaît aussi dans le champ français comme indéniable source d'inspiration pour bien des penseurs désireux d'appréhender la subjectivité comme dessaisissement ou déprise, de la penser donc au prisme de l'événement. Par exemple, si Levinas a critiqué la psychanalyse freudienne lui reprochant de méconnaître le sens radical de la subjectivité et de la conscience ${ }^{25}$ et mettant en cause le sort qu'elle réserve à la conscience, il a aussi pu affirmer avec elle que la conscience n'épuise pas la notion de subjectivité. Il a ainsi indiqué en quoi sa pensée peut rejoindre la psychanalyse, en particulier touchant « une subjectivité qui se poserait, peut-être en se dé-posant, tel un roi qui renonce à son royaume et à sa royauté ${ }^{26}$. Dans le même sens, il a insisté sur la proximité existant entre l'intentionnalité qui lie la

\footnotetext{
${ }^{21}$ Ibid., p. 157.

${ }^{22}$ LyOTARD Jean-François, « Voix », p. 129-153, Lectures d'enfance, Paris, Galilée, Débats, 1991, p. 144 et 148.

${ }^{23}$ Le 4 octobre 1907, il note : «Pas bien reproduit ; beaucoup est manqué, s'est estompé, parmi les beautés singulières du cas » (p. 17-63). Et les 22 et 23 novembre, il explose, répondant à un affect par un affect : « Autre irruption d'une idée des plus horribles... » et « la séance suivante est remplie par le transfert le plus épouvantable... » (p. 64-65). Voir Sigmund Freud, L'Homme aux rats, Journal d'une analyse, Paris, PUF, Bibliothèque de psychanalyse, 1974.

${ }^{24}$ LYOTARD Jean-François, « Voix », op. cit., p. 148.

${ }^{25}$ LEVINAS Emmanuel, Entre nous. Essais sur le penser-à-l'autre, Paris, Grasset, Figures, 1991, p. 36-37. Et « Quelques vues talmudiques sur le rêve », p. 114-128, La psychanalyse est-elle une histoire juive ? Colloque de Montpellier, 1980, A. \& J.-J. Rassial (dir.), Paris, Seuil, 1981, p. 127.

${ }^{26}$ Ibid., p. 114.
} 
pensée à un implicite et la psychanalyse ${ }^{27}$. Ou plutôt, il a contribué à re-thématiser l'intentionnalité à partir de ses marges ou de ses angles morts, prenant à revers certains travaux qui doutaient de la possibilité de penser le dessaisissement de l'intelligence par l'inconscient ou l'expérience de l'œuvre d'art dans les termes de la phénoménologie ${ }^{28}$.

C'est pourquoi une série de travaux ont conduit à confronter, comparer ou rapprocher les pensées lacanienne et lévinassienne ${ }^{29}$, dans l'idée que l'affirmation de l'existence du sujet est solidaire de l'épreuve de sa possible disparition, soit d'un événement traumatisant. Pour penser le tissu dramatique de la vie et de l'identité du sujet, l'analyse mêle le motif lévinassien de l'interpellation par autrui du sujet et la conception psychanalytique du trauma. Une complicité se noue entre les trois références, si bien que «[les] considérations de Lacan sont inspirées par un souci qui est aussi le nôtre, c'est-à-dire de comprendre comment quelque chose de totalement étranger peut se donner ou plutôt s'imposer à un sujet, et comment ce sujet peut se rapporter à cet impossible $»^{30}$. D'une façon générale, la psychanalyse lacanienne possède de grands attraits pour des penseurs qui aspiraient à rompre avec le surplomb idéaliste d'une philosophie solitaire et désireux de rompre avec un concept idéaliste du sujet en pensant la subjectivité comme événement ou dessaisissement ${ }^{31}$.

On pourrait montrer par exemple que l'analyse de ce qui constitue, dans la théorie politique d'Ernesto Laclau, l'événement fondamental, à savoir la décision (et le problème de décider en terrain indécidable) ne peut être conduite que grâce à une théorie du sujet, nourrie et inspirée par la psychanalyse lacanienne. Ce sujet dont la structure ne décide pas, qui doit s'autodéterminer, qui constitue ainsi le point aveugle dans la structure permet de penser l'événement de la décision que Laclau nomme «folie de la décision » dans la mesure où il s'agit de tirer quelque chose de soi, d'on ne sait où, quelque chose qui n'est jamais donné, une supplémentarités ${ }^{32}$, pour pouvoir agir, décider.

Pourtant, on a surtout eu recours à la psychanalyse pour former une pensée de l'effraction, en raison de la conception du sexuel comme puissance disruptive dont celle-ci est porteuse. Il faut rappeler d'abord combien la libido diffère d'un désir qui atteint son but et par lequel le sujet se retrouve. En effet, la réalité des processus primaires est celle d'une énergie libre qui poursuit une décharge et qui n'est pas fixée, investie, dans une association stable. Il s'agit d'un désir qui ne s'investit pas mais qui bouleverse, fait événement. La pulsion de mort pourrait être du côté de cette énergie déliée et déliante. Lyotard en particulier a thématisé cette événementialité du désir libidinal, dégageant chez Freud une oscillation entre deux conceptions du désir : le "désir-Wunsch», qui implique une dynamique, une négativité, une téléologie au sens où celui-ci tend à recouvrer l'objet perdu, et le « désir-libido » qui possède deux régimes, dans lesquels prime l'afflux et la décharge - répétitifs et non finalisés - d'énergie, énergie qui peut être "détraquante ", " désordonnée ", " déconstruisante, et même "mort » ${ }^{33}$. Il est clair qu'en étant travaillé par un tel désir-libido, la subjectivité se trouve dessaisie ou détrônée ; elle abdique, pour reprendre l'image de Levinas.

L'idée de trauma ne nous semble pas disparaître des textes freudiens, à la fin des années 1890, avec la découverte du fantasme, quoique le modèle étiologique reste oscillant, comme en témoigne cette déclaration de 1912 :

\footnotetext{
${ }^{27}$ LeVINAS Emmanuel, En découvrant l'existence avec Husserl et Heidegger, Paris, Vrin, Bibliothèque d'histoire de la philosophie, 1988, p. 130. Voir Simon Critchley, «Le traumatisme originel - Levinas avec la psychanalyse », p. 165174, Emmanuel Levinas, Rue Descartes, Paris, PUF, Quadrige, Essais débats, 2006.

${ }^{28}$ Dans Discours, figure, par exemple, Lyotard, menant une critique de la philosophie esthétique de Merleau-Ponty, en vient à soutenir que l'intentionnalité ne peut s'approcher du figural qui est toujours désir, soit inconscient ou expression, c'est-à-dire aussi chose inquiétante et informe. Plus encore, l'intentionnalité phénoménologique lui semble tributaire d'une structure d'adresse qui ne rendrait pas compte de l'absolument inadressé qui prime dans le figural, l'intensité, ou l'affect.

${ }^{29}$ Voir Assoun Paul-Laurent, Le sujet et l'Autre chez Levinas et Lacan, Rue Descartes, Paris, Albin Michel, $n^{\circ} 7, \mathrm{p}$. 124-145, juin 1993.

${ }^{30}$ BERNET Rudolf, Conscience et existence. Perspectives phénoménologiques, op. cit., p. 275.

${ }^{31}$ Voir PAGÈs Claire, "Le Sujet divisé à l'épreuve de la différence », p. 61-99, in Penser avec Lacan. Nouvelles lectures, G. F. Duportail (dir.), Hermann, « Philosophie », avril 2014.

${ }^{32}$ LACLAU Ernesto, «Déconstruction, pragmatisme, hégémonie », p. 99-136, Déconstruction et pragmatisme, Les Solitaires Intempestifs, Expériences philosophiques, 2010, p. 114. Voir PAGÈS Claire, « Politique et postmodernité : entre micrologies et logiques hégémoniques. J.-F. Lyotard et E. Laclau », p. 95-113, in Morales et politiques postmodernes, M. Kowalska (dir.), Peter Lang, 2014.

${ }^{33}$ LYOTARD Jean-François, «La peinture comme dispositif libidinal (genre parlé improvisé) », p. 227-267, Des dispositifs pulsionnels, Christian Bourgois, 1980, p. 229-230.
} 
Les névrosés vivent dans un monde particulier dans lequel, comme je l'ai exprimé ailleurs, a seule cours la «monnaie névrotique », c'est-à-dire que chez eux est seul efficient ce qui est pensé intensément, représenté avec affect, tandis que la concordance de cela avec la réalité externe est accessoire. L'hystérique répète dans ses accès et fixe dans ses symptômes des expériences vécues qui ne se sont déroulées que dans sa fantaisie, remontant il est vrai, en dernière analyse, à des événements effectifs ou ayant été édifiées à partir de ceux-ci. ${ }^{34}$

Si la théorie de la séduction est abandonnée avec la généralité de la perversion du père pendant l'année 1897 pour ouvrir la voie progressivement à une théorie du fantasme, théorie de la causalité par l'image inconsciente, par la trace mnésique déjà inscrite, mais aussi à l'hypothèse de la constitution (le développement sexuel comme tributaire de la constitution sexuelle dans les Trois essais), n'est pas abandonné au contraire ce qui la motivait et qui tenait à la nature et à l'effet traumatique de la sexualité. Même en reconnaissant l'illusion d'une sexualité introduite du dehors chez l'enfant « innocent » par l'adulte, la théorie de la séduction reste un modèle théorique fascinant. Ce modèle traumatique impose de tenir ensemble l'exigence d'une effraction, d'une consistance d'un dehors et d'un dedans, dans un lieu non encore sexuel, et celle d'un lieu pourtant sexuel pour pouvoir être réceptif et éveillé par l'effraction. Il suppose un sujet sexuel pour moitié comme signification subie - étrangère, traumatique - dans la passivité d'une effraction et de l'autre un minima d'activité et de constitution qui permet la formation d'une expérience : « un sujet d'avant le sujet et recevant son être, son être sexuel, d'un extérieur d'avant la distinction intérieurextérieur $»^{35}$. Nous semble décisive la dimension d'effraction d'une part - en cela le français " après-coup » est très évocateur, car il suppose un " coup » même perçu avec retard dans son caractère traumatique. C'est pourquoi Laplanche parle de bombe à retardement pour illustrer ce point : «Le premier souvenir est comme une bombe à retardement qui serait déclenchée secondairement par une mise à feu ${ }^{36}$.

D'autre part, est décisive la dimension dérangeante du sexuel, qui nomme chez Freud la force de ce qui est désir déplaisant, désagréable, non policé, peu présentable. C'est pourquoi le processus de sublimation comme destin de la pulsion sexuelle contribue à soutenir la culture et la formation du lien social. La théorie de la séduction généralisée peut s'ancrer dans une compréhension de la séduction distincte des actes effectifs d'atteinte et d'outrage mais qui envisage les excitations sexuelles dans les soins donnés au corps de l'enfant mais aussi liés aux fantasmes inconscients de l'adulte (c'est le concept de «message » chez Laplanche). Le sexuel reste introduit du dehors, par effraction, sans que cela implique un attentat ou une agression ${ }^{37}$.

C'est pourquoi Lyotard se sert du concept freudien de la sexualité - infantile en particulier - pour penser à la fin des années 1980 et dans les années 1990, ses notions de touche, d'enfance, qui impliquent une effraction, non-préparée, chez un sujet sans défense. Déjà, dans Discours, figure, en 1971, il retenait en particulier des analyses freudiennes cette distinction entre processus primaires et processus secondaires, accentuant l'opposition entre principe de plaisir et principe de réalité, concevant la figure comme mouvement de l'énergie libre, comme ce qui n'est pas fixé, arrêté, investi dans des associations fixes, fussent-elles énergétiques. D'où son intérêt aussi pour la logique de la pulsion de mort, qui est énergie déliée et déliante. Le figural ne se comprend ainsi que dans un espace où l'énergie circule librement. De même que l'énergie libre qui anime les processus primaires poursuit une décharge, la figure est, tout comme le symptôme, ce qui fait événement, ce qui, venant d'un ordre, vient en bouleverser un autre. Le langage articulé est alors pris pour analogon du refoulement, comme ce qui a tendance à opérer un rejet de la figuralité. Ce qu'une image a de figé et qui la rend reconnaissable est la concession que l'ordre du discours, le principe de réalité, impose au figural. Freud aurait donc saisi la «connivence » du désir avec le figural et ainsi ouvert la voie à l'écoute du figural. Lyotard lit donc l'Interprétation du rêve en supposant que le désir avec ses processus primaires dont parle Freud est puissance de figuration, écrivant de Freud qu'il a saisi une vérité non discursive, une vérité qui relève du coup de force. Celle-ci froisse, plie, et détonne : "la vérité se

\footnotetext{
${ }^{34}$ FreUd, « Totem et Tabou, Quelques concordances dans la vie d'âme des sauvages et des névrosés », 1912-1913, p. 189-385, Euvres complètes, Psychanalyse, volume XI, Paris, PUF, 1998, 2e éd. 2005, p. 296.

${ }^{35}$ LaPlanche Jean \& PONTALIS Jean-Bertrand, Fantasme originaire, Fantasme des origines, Origine du fantasme, Paris, Hachette, Textes du XXe siècle, 1985, p. 28.

${ }^{36}$ « Après-coup », Dictionnaire international de la psychanalyse, Paris, Hachette Littératures, Grand Pluriel, 2005.

${ }^{37}$ Le travail de Christophe Dejours a permis de souligner cette dimension de la notion freudienne de sexualité, s'inscrivant dans la lignée de Laplanche qui fait de la sexualité chez Freud une anthropologie, signifiant que le sexuel n'est pas un objet parmi d'autres mais structure l'ensemble du champ humain. Voir DEJOURS Christophe, Travail vivant, 1 : Sexualité et travail, Paris, Payot, 2009, p. 85 sq.
} 
manifeste comme une aberration à l'aune de la signification et du savoir. Elle détonne. Détonner dans le discours c'est déconstruire son ordre $»^{38}$. Le désir dans le processus primaire, mais aussi le figural violentent un contenu : «Le désir ne parle pas, il violente l'ordre de la parole » ${ }^{39}$.

La difficulté tient au fait que le fantasme tient en bride le processus primaire, le fixe et l'arrête dans une forme, l'attache à un objet. Le fantasme bloque et aplatit la différence ou l'événement en particulier dans une forme répétitive ou itérative. La déconstruction de la bonne forme et l'activité critique opèrent grâce à l'énergie des processus primaires, si le fantasme opère comme un filet qui lie le désir, l'événement du figural, à une forme et direction définies. La fonction critique de l'œuvre tient alors au fait, explique Discours, figure, que les opérations primaires fonctionnent pour elles-mêmes et pas comme moyen, en vue d'une réalisation dans une certaine forme. Elle consiste à donner à voir dans l'œuvre les opérations du désir. L'esthétique psychanalytique serait alors coupable de confondre souvent œuvre et hallucination faisant des productions artistiques des espaces de leurre, des formes sur l'existence desquelles on se méprend ${ }^{40}$. Est révolutionnaire l'ordre du figural, comme le nomme Lyotard à la fin des années 1960 et au début des années 1970, ou son événement - dérangement des processus secondaires par les processus primaires et irruption des formes venant de l'inconscient au sein des processus secondaires - et de démasquer toutes les tentatives visant à le fixer en une «écriture » ou un « écran » (tentatives qui désamorcent l'événementialité du désir). L'œuvre est critique, si elle s'ouvre à l'ordre du figural, celui de l'événement. Bien sûr, c'est à une autre figure de l'événement que nous sommes ici confrontés : ce n'est plus le réel qui se trouve ici rencontré de façon brutale ou traumatique, mais le désir lui-même qui affecte et bouleverse son propre sujet de façon intempestive.

Ainsi, la tendance freudienne au discours de vérité, ce qui inscrit celle-ci dans la ratio occidentale, est-elle perturbée par une place ménagée à l'événement, et ce en particulier grâce à l'idée de pulsions de mort : « les pulsions de mort, dernier événement, marqueront dans la théorie la place de l'indévisageable et de l'insignifiable, la limite de la représentation et de la théorie ${ }^{41}$. La mort dont il s'agit relève donc bien de l'événement du figural. Il est intéressant que Lyotard lie la pensée de l'événement et le caractère hypothétique ou spéculatif de l'idée freudienne de pulsion de mort ${ }^{42}$. Le second dualisme pulsionnel a l'audace de reposer sur un événement, celui de la division des pulsions et de s'affranchir de sa fonction référentielle ${ }^{43}$. Tout cela aboutit à un «effet d'incertitude » qui empêche de décider du vrai et du faux... Cette autre modalité du discours où le vrai devient «une affaire de style ${ }^{44}$, ce discours du style qui se distingue du dire-le-vrai scientifique est solidaire d'une impassibilité nommée aussi apathie. Freud aurait comme malgré lui résisté au "terrorisme théorique », dont il se prétend pourtant le commanditaire, et à ses prêtres. L'apathie semble alors identifiée comme forme de passibilité à l'événement, identifiée avant l'heure comme passibilité au il arrive.

Dans les analyses qui précèdent, l'événement est recherché dans le champ de la subjectivité, du côté d'une subjectivité revisitée ou déconstruite. Celle-ci est dessaisie, déposée, ou transie. On retrouve la dimension d'effraction, puisqu'elle est secouée par de l'autre (altérité d'autrui ou du sexuel par exemple). Ce régime de l'événement nomme encore ce qui vient déconstruire un ordre, le bouleverser. C'est ce qui vient perturber la bonne forme. D'où l'intérêt pour le motif du symptôme que Lyotard compare à la figure qui fait événement et que Derrida, nous y reviendrons, définira comme un des sens de l'événement. Pourquoi la psychanalyse s'est-elle révélée précieuse pour peser la déconstruction de la représentation et du sujet ? En raison, d'une façon générale, du caractère vexatoire, dit Freud, d'une théorie qui constitue la troisième blessure, la plus profonde d'ailleurs, pour le narcissisme humain. En raison, plus spécifiquement, de la pensée du libidinal ou du processus secondaire et du sexuel comme effraction. En raison, enfin, de l'idée de pulsion de mort.

\footnotetext{
${ }^{38}$ LyOTARD Jean-François, Discours, figure, Paris, Klincksieck, 1985, p. 17.

${ }^{39}$ Ibid., p. 244.

${ }^{40}$ LYOTARD Jean-François, « Notes sur la fonction critique de l'œuvre », Dérive à partir de Marx et Freud, Paris, 10/18, 1973, p. 232.

${ }^{41}$ LyOTARD Jean-François, « Edipe juif », p. 167-188, Dérive à partir de Marx et Freud, op. cit., p. 174.

${ }^{42}$ LYOTARD Jean-François, Discours, figure, op. cit., p. 18-19.

${ }^{43}$ LyOTARD Jean-François, «L'apathie théorique », p. 254-265, Critique n³33, février 1975, La psychanalyse vue du dehors (I), Minuit, p. 263 ; p. 256-257.

${ }^{44}$ Ibid., p. 254.
} 
(3) Cette incertitude quant à l'usage qu'il faut faire de la psychanalyse pour penser l'événement prend sa source dans l'ambiguïté profonde de cette interprétation des destins psychiques inconscients, partagée entre la prise en compte de la dimension d'effraction attachée à la sexualité et l'idée de développements pulsionnels endogènes et d'une logique autonome de la réalité psychique, logique de l'image ou du fantasme. La pensée de l'CEdipe témoigne de cette oscillation, cette effraction du sexuel devenant peu à peu structure ou du moins loi, comme l'indique cette lettre bien connue de Freud à Fliess : "Chez moi aussi j'ai trouvé le sentiment amoureux pour la mère et la jalousie envers le père, et je les considère maintenant comme un événement général de la prime enfance [ein allgemeines Ereignis früher Kindheit] ${ }^{45}$. On peut en effet interroger la consistance d'un événement devenu ainsi « général ».

Mieux que personne, Laplanche et Pontalis, dans le texte de 1968, Fantasme originaire, fantasmes des origines, origines du fantasme, ont dégagé les ressorts et les étapes de cette pensée oscillante. Celle-ci fait droit et place à la fonction étiologique du traumatisme, qu'on retrouve dans la conception du sexuel (« il est dans la nature même de la sexualité d'avoir un effet traumatique... » ${ }^{46}$ ), raison pour laquelle, il y a d'après eux quelque chose de profond dans la théorie de la séduction, par-delà ses errements, son abandon et son désaveu par Freud. On sait que Laplanche travaillera alors à formuler une théorie de la séduction généralisée. Mais cette pensée freudienne montre également combien est vaine la recherche de l'événement premier, celle du trauma originaire, si bien que, si événement il y a, sa texture de fantasme, nous y reviendrons, l'inscrit plutôt dans une logique de répétition sans événement inaugural. Les deux auteurs dégagent également ce qui chez Freud contrecarre et menace de recouvrir toute pensée de l'événement psychique, à savoir la thématisation des constitutions, des prédispositions (nouveau visage donné à l'ancienne disposition héréditaire): "Si l'événement se dérobe, alors l'autre terme de l'alternative - la constitution - est réhabilitée $»^{47}$.

Cette place très contrastée ménagée à l'événementialité se retrouve dans la conception de la cure. Andrée Green l'a souligné, analysant la situation particulière de la psychanalyse entre historicisme et structuralisme. La recherche dans la cure d'un fondement historique du fait de la répétition, celle de l'enfance, l'insistance sur la régression, les stades, la dimension de développement psychique, quoique non linéaire et distinct d'une évolution naturelle, témoigne d'une centralité de l'événement. Celle-ci est pourtant contrecarrée par le doute qui plane sur la réalité de ce passé retrouvé, dont la quête se voit préférer « la traduction du manifeste au latent à l'aide des lois du symbolique $»^{48}$ (étude de la relation entre les signifiants, des substitutions entre les signifiants, etc.). En réalité, de même que l'alternative théorique «constitution - événement » est un leurre, comme l'ont montré Laplanche et Pontalis, la pratique, la cure, n'est pas le plus souvent clivée de la sorte entre la recherche de l'événement et son abandon, sauf dans des formes limites (dans la psychopathologie néojacksonienne d'Henri Ey par exemple d'après Green), mais elle mêle absolument analyse structurale des signifiants avec l'exploration et la reconstruction anamnésique du passé.

Le statut de l'événement s'en trouve, en tout état de cause, redéfini. L'événement dont la cure lève le refoulement ne peut plus se confondre jamais avec le fait empirique, car il possède justement le plus souvent une réalité psychique et discursive (et non uniquement matérielle). É. Benveniste l'a clairement formulé :

[...] le fait biographique ne peut porter à lui seul le poids d'une connexion causale. [...] Supposons même que, dans un univers utopique, l'analyste puisse retrouver, en témoignages objectifs, la trace de tous les événements qui composent la biographie du patient, il en tirerait encore peu de chose, et non, sauf accident heureux, l'essentiel. Car s'il a besoin que le patient lui raconte tout et même qu'il s'exprime au hasard et sans propos défini, ce n'est pas pour retrouver un fait empirique qui n'aura été enregistré nulle part que dans la mémoire du patient : c'est que les événements empiriques n'ont de réalité pour l'analyste que dans et par le «discours » qui leur confère l'authenticité de l'expérience, sans égard à leur réalité historique, et même (faut-il dire : surtout) si le discours élude, transpose ou invente la biographie que le

\footnotetext{
${ }^{45}$ FreUd, Lettre du 15 octobre 1897, Lettres à Wilhelm Fliess 1887-1902, Paris, PUF, 2006, p. 344-345.

${ }^{46}$ LAPlanche J. \& PONTAlis J.-B., Fantasme originaire..., op. cit., p. 22-23.

${ }^{47}$ Ibid., p. 31.

${ }^{48}$ GREEN André, « La psychanalyse devant l'opposition de l'histoire et de la structure », p. 649-662, Minuit, Critique $1963 / 07$, p. 654.
} 
sujet se donne. Précisément parce que l'analyste veut dévoiler les motivations plutôt que reconnaître les événements. $^{49}$

Si l'analyse ne saurait se confondre avec la quête d'un donné historique (un événement) enfoui, elle n'en recherche pas moins ce qui faisant événement a été refoulé, quoique cet événement se distingue le plus souvent d'un fait (traumatisme par exemple). Aussi la pensée psychanalytique de l'événement a pu être recherchée, on l'a dit, du côté de sa conception du sexuel ou des processus primaires comme ce qui fait effraction (dans une forme d'enfance, d'infantia, quelle qu'elle soit). C'est pourquoi, selon Lyotard, la psychanalyse freudienne aurait anticipé cette forme d'événement qu'est la touche ${ }^{50}$.

On comprend mieux maintenant que la psychanalyse ait pu constituer une référence si ambivalente pour la pensée française contemporaine de l'événement :

1. Si on met l'accent, dans la théorie psychanalytique, sur le poids du symbolique, sur les phénomènes endogènes, sur la logique autonome du phantasme, sur ces images qui ont le pouvoir de nous affecter sans être le reflet d'un quelconque réel, sur les structures, les complexes, celle-ci semble peu à même de fournir des outils pour une pensée de l'événement, du traumatisme ou de l'émergence du singulier. Par ailleurs, et pour les mêmes raisons, elle a pu séduire aussi des penseurs qui récusaient la centralité de l'événement, qui, à rebours de l'historicisme dominant, ont tenté, après guerre, en particulier en France, de faire valoir les logiques structurelles. Il suffit de penser ici à l'intérêt par exemple de Lévi-Strauss pour l'idée freudienne de la constitution inconsciente du sens, qui va de pair chez Freud avec l'«atemporalité [Zeitlosigkeit] $»$ de nos processus inconscients $»^{51}$. Si l'inconscient est atemporel, et si seuls les phénomènes de conscience se trouvent pris dans l'histoire, la psychanalyse freudienne contribue à étayer l'idée que les processus déterminants sont anhistoriques quand seuls les phénomènes secondaires engendrés connaissent le régime - bien apparent - de l'événement. Lévi-Strauss récusera par ailleurs ce qui semble faire pencher la théorie psychanalytique du côté de l'événement ou de l'histoire, reprochant à Freud d'avoir pris l'inceste pour un événement réel. Ce que Freud a tenu à tort pour origine historique est structure de toute société, événement qui tient son efficacité de n'en être justement pas un, de ne s'être pas réalisé : «Les satisfactions symboliques dans lesquelles s'épanche, selon Freud, le regret de l'inceste, ne constituent donc pas la commémoration d'un événement. Elles sont autre chose, et plus que cela : l'expression permanente d'un désir de désordre, ou plutôt de contrordre $»^{52}$.

2. À l'inverse, si l'on se concentre sur la pensée psychanalytique du trauma, si l'on met en avant le caractère disruptif du sexuel et des processus primaires, celle-ci devient un allié de choix pour repenser la subjectivité en termes d'événements ou à l'aune d'une événementialité enfin reconnue. Cette même théorie psychanalytique est alors récusée par ceux qui tentent de circonscrire, ou de penser différemment cette accidentalité. Par exemple, les penseurs de la résilience ont très tôt mis en cause la pensée psychanalytique « événementialiste» du traumatisme ${ }^{53}$. La prise en compte de la capacité de sortir vainqueur et grandi d'une épreuve représentant un traumatisme est censée pendre le contre-pied du modèle psychanalytique de la vulnérabilité, en lui opposant la possibilité de la métamorphose, l'importance de la dimension adaptative et évolutive du moi, les puissances de protection des sujets et de leur environnement. En mettant l'accent sur les concepts de traumatisme psychique et de trouble réactionnel, la psychanalyse freudienne serait dans l'impossibilité de saisir la capacité d'un sujet à s'adapter face au danger et à se développer normalement après une épreuve. Dans la théorie de la résilience, le syndrome post-traumatique, les phénomènes de répétition émanent donc du milieu du sujet et non de sa psyché : « Nous nous trompons de malade. Ce n'est pas tant sur le blessé qu'il faut agir afin qu'il souffre moins, c'est surtout sur la culture ${ }^{54}$. C'est pourquoi l'oxymoron élaboré par le résilient est pensé sur le modèle de la perle fabriquée par l'huître en réaction à une

\footnotetext{
${ }^{49}$ BENVEniste Émile, « Remarques sur la fonction du langage dans la découverte freudienne », p. 75-87, Problèmes de linguistique générale I, Paris, Gallimard, Tel, 1966, p. 76-77.

${ }^{50}$ Voir LYOTARD Jean-François, La Confession d'Augustin, Paris, Galilée, Incises, 1998, p. 79.

${ }^{51}$ FrEUD, « Sur l'engagement du traitement », 1913, p. 161-184, CEuvres Complètes XII, Paris, PUF, 2005, p. 170. Voir Gesammelte Werke VIII, Frankfurt am Main, S. Fischer Verlag, 1990, p. 462. Et FrEUD, « Au-delà du principe de plaisir », 1920, p. 273-338, Euvres Complètes XV, op. cit., p. 299. Voir Gesammelte Werke XIII, Frankfurt am Main, S. Fischer Verlag, 1940, 1987, p. 28.

${ }^{52}$ LÉVI-STRAUSS Claude, Les Structures élémentaires de la parenté, La Haye, Mouton, 1949/1967, p. 563.

${ }^{53}$ Les recherches sur la résilience démontreraient en particulier la fausseté des arguments psychanalytiques relatifs au caractère absolument négatif de la carence maternelle précoce sur le développement des sujets et sur le pont établi entre traumatisme et névrose.

${ }^{54}$ CYRULNIK Boris, Un merveilleux malheur, Odile Jacob, poches, 2002, p. 174.
} 
agression. Il est intéressant que soit ainsi visée la fausse extériorité de l'événement psychique. L'événement est manqué car le modèle du trauma l'intériorise et le rend ainsi insurmontable ${ }^{55}$.

(4) On dégagera brièvement avant de conclure quelques-uns des contributions possibles de la théorie psychanalytique, freudienne en particulier, à une pensée contemporaine de l'événement.

Le premier apport tient à la révision que celle-ci implique du rapport entre temps et événement et à la possibilité de les penser séparément. En effet, comme on l'a dit, l'inconscient ignore le temps, ses processus sont atemporels. Les événements psychiques ne sont pas ainsi pensables et situables dans le temps à la manière des événements historiques, si bien que deviennent contemporains des contenus d'époques distinctes. Si bien également que peut continuer à agir comme événement une représentation très ancienne protégée de l'érosion du temps par le refoulement et qui serait parvenue à s'adjoindre à nouveau des forces (une énergie d'investissement), en proliférant, comme dit Freud, pour ainsi dire, "dans l'obscur ${ }^{56}$. Cette atemporalité disjoint donc événementialité et actualité. C'est ce qui a pu, par exemple, intéresser Derrida, comme matrice d'une pensée de la hantise ou de la revenance ${ }^{57}$.

La théorie psychanalytique permet deuxièmement de revenir sur l'association traditionnelle entre événement et extériorité et sur l'opposition tout aussi classique entre événement et intériorité, comme si l'événement était nécessairement ce qui ébranle, du dehors, un dedans. Dans la pensée psychanalytique de l'événement psychique, c'est en effet davantage le psychisme qui fait l'événement. Il n'y a pas de proportion entre un fait empirique vécu et son destin et traitement psychique. Bien plus, la psyché forge ses propres événements, les fantasmes, ayant ainsi la puissance de s'auto-affecter, raison pour laquelle Freud affirme que les hystériques souffrent le plus souvent non pas d'un traumatisme suivant une agression, mais de réminiscences. Si ces réminiscences peuvent entretenir un rapport avec un événement historique, celui-ci n'est pas de l'ordre de la causation simple et directe ${ }^{58}$. Cette absence d'extériorité de l'événement est au cœur de la théorie de l'Unheimlich, cette inquiétante étrangeté qui caractérise ce qu'il y a de plus familier, l'intériorité, théorie qui inspire, comme on le sait, les idées derridiennes du double et de l'indécidable.

Ces concepts psychanalytiques ont alimenté la pensée derridienne de l'événement de l'hospitalité. La saisie de l'événement, quoi qu'on y fasse, suppose son itérabilité dans un dire qui emporte son unicité : «la venue de l'événement inaugural - ne peut être accueillie que comme retour, revenance, revenance spectrale $»^{59}$. Or, pour penser cette hantise qui est, dit Derrida, « la structure spectrale de cette expérience de l'événement ${ }^{60}$, la référence psychanalytique s'impose. Celle-ci mêle en effet pensée de l'événement intérieur dissimulé (et tout événement est secret, souligne Derrida) et idée d'effraction. C'est pourquoi le symptôme, écrit-il, est une signification de l'événement car personne, aucun sujet conscient, ne le maîtrise, ne peut se l'approprier. Le symptôme est, rappelle Derrida, ce qui tombe, et pour cette raison la vérité de l'événement de l'arrivant. En effet, l'arrivant absolu est quelqu'un dont la venue inopinée ou la « visitation »

\footnotetext{
55 On pourrait montrer comment d'une façon très différente l'accent qui serait mis par la psychanalyse sur les événements de la vie venant former une biographie - et même un destin - l'empêcherait de saisir une causalité radicalement non événementielle. Ainsi la psychanalyse alimenterait ce que Pierre Bourdieu nomme l'illusion biographique, occultant ce faisant les mécanismes sociaux qui autorisent et conditionnent l'expérience de la vie comme unité et totalité, dissimulant que cette identité personnelle pratique vient de l'habitus comme principe actif, principalement socialement assignée par le nom propre, qui constitue l'institution de totalisation et d'unification du moi la plus évidente... Cette critique du biographisme s'accompagne par ailleurs, chez Bourdieu, de l'idée que l'inconscient psychique constitue un oubli, voir un déni de l'histoire, signe qu'on peut se focaliser sur des événements sans se tenir pour autant dans une quelconque historicité.

${ }_{56}$ FreUD, «Le refoulement », 1915, p. 189-203, Euvres Complètes XIII, Paris, Puf, 1993, p. 194. Voir Gesammelte Werke X, Frankfurt am Main, S. Fischer Verlag, 1973, p. 251.

57 Voir FreUd, «L'inquiétant», 1919, p. 147-188, in OC XV, et DERRIDA, « Spéculer - Sur “Freud" ", in Carte postale, op. cit., p. 362-363.

${ }^{58}$ LAPlAnche Jean, Problématique V, Le baquet, transcendance du transfert, Paris, PUF, 1987, p. 128: «Les signifiants, pour autant qu'on puisse les imaginer dans l'inconscient, n'y constituent rien qui ressemble à une structure de code. Ils ne renvoient qu'à eux-mêmes, ou peut-être, par une sorte de réminiscence vague mais qui n'est d'aucune façon souvenir, à l'événement historique dont ils sont extraits ».

${ }^{59}$ DERRIDA Jacques, « Une certaine possibilité impossible de dire l'événement », p. 79-112, J. Derrida \& A. Nouss \& G. Soussana, Dire l'événement, est-ce possible? Séminaire de Montréal, pour Jacques Derrida, Paris, L'Harmattan, Esthétiques, 2001, p. 98.

${ }^{60}$ Ibid., p. 99.
} 
est une telle irruption que je ne suis pas préparé à (ni capable de) l'accueillir: "l'événement, comme l'arrivant, c'est ce qui verticalement me tombe dessus, sans que je puisse le voir venir ${ }^{61}$. En même temps, cette passibilité à l'irruption est conditionnée par une familiarité avec l'étrangeté intérieure. Plus profondément, explique Derrida dans sa contribution au Manifeste pour l'hospitalité, la condition de l'hospitalité pure qu'il nomme " événement sans grammaire préalable » ${ }^{62}$ est d'être d'abord hospitalier avec sa « société interne " ${ }^{63}$, avec l'étranger en soi, avec sa propre Unheimlichkeit. C'est la pensée nourrie de psychanalyse freudienne qui permet alors d'entendre cela : «Il n'y a pas d'hospitalité sans turbulence et sans risque de transformation, de séisme, sans exposer son propre chez soi à la turbulence $»^{64}$.

Nous dirions volontiers, troisièmement, qu'un des concepts les plus riches pour une pensée de l'événement demeure celui d'après-coup (nachträglich; Nachträglichkeit). Pour comprendre l'intérêt que constitue cette notion, commençons par la distinguer de l'abréaction ou de l'action différée. Il ne s'agit pas de signaler simplement l'existence d'un délai entre action et réaction, événement et effets, excitations et réponse/décharge ${ }^{65}$. L'après-coup désigne plutôt la dualité de l'événement ou le fait que, psychiquement, très souvent, un premier événement devient tel à la faveur d'un second ou d'une seconde série d'événements. C'est le remaniement dont sont l'objet les événements du passé après-coup qui leur conférera un sens, un caractère pathogène, etc. Plus précisément, est réélaboré un événement, un fait qui n'avait pu prendre sens pour le sujet au moment de sa survenue. Ce remaniement est occasionné par d'autres événements et situations. C'est ce qui reste vrai de la théorie de la séduction sexuelle et sert à comprendre la vie sexuelle même :

D’une part - premier temps - la sexualité fait littéralement irruption du dehors, pénétrant par effraction dans un «monde de l'enfance » présumé innocent où elle vient s'enkyster comme un événement brut sans provoquer de réaction de défense : l'événement n'est pas par lui-même pathogène. D'autre part, au deuxième temps, la poussé pubertaire ayant déclenché l'éveil physiologique de la sexualité, il y a production de déplaisir et l'origine de ce déplaisir est cherchée dans le souvenir de l'événement premier, événement du dehors mué en événement du dedans, " corps étranger » interne qui cette fois fait irruption du sein même du sujet. ${ }^{66}$

Cette idée d'après-coup structure la théorie du refoulement. La théorie de l'après-coup témoigne ainsi du fait que l'histoire du sujet n'est pas linéaire, se distinguant absolument d'un schéma dans lequel des événements passés produiraient une action dans le présent. La représentation d'événements définissant un avant et un après s'en trouve également bousculée. Lyotard, en particulier, s'est nourri de cette pensée freudienne de l'après coup et du retard pour penser l'événement de l'enfance qu'il nommera aussi infantia, phonè, touche, etc. :

Il n'y a pas de présentation dont on puisse dire qu'elle est une «première ». L'apparition de ceci réitère cela. Ce n'est pas qu'elle redouble la même chose ou répète la même scène. C'est au sens où Freud entend nachträglich. Le premier coup nous a touché l'âme trop tôt, le second l'aura touchée trop tard. La première fois est comme une pensée déjà là, mais pas encore pensée ; la seconde est cet impensé qui revient et demande à être pensé, mais quand la première n'y est plus. ${ }^{67}$

Enfin, quatrièmement, la pensée de l'événement psychique permet de dissocier événement et origine. Les événements psychiques pour la plupart sont en effet sans origine. La recherche d'un premier terme, trauma, premier souvenir, fait long feu, se révèle vaine ; ce qui ne signifie absolument pas que rien n'est arrivé. Du moins, ne trouve-t-on pas souvent de premier terme à la répétition. En le comprenant, Freud abandonne la théorie de la séduction sexuelle: "la recherche de l'événement premier a abouti à une impasse... ${ }^{68}$, commentent Laplanche et Pontalis. C'est d'ailleurs ce qui intéressa Deleuze dans la pensée freudienne de la répétition, l'événement sans origine : «Un moment décisif de la psychanalyse fut celui où Freud renonça sur

\footnotetext{
${ }^{61}$ Ibid., p. 97.

${ }^{62}$ SefFAhi M. (dir.), Autour de Jacques Derrida, Manifeste pour l'hospitalité, Grigny, Paroles d'aube, 1999 , p. 98.

${ }^{63}$ Ibid., p. 139.

${ }^{64}$ Ibid., p. 143.

${ }^{65}$ LAPLANCHE Jean et PONTALIS Jean-Bertrand, Vocabulaire de la psychanalyse, Paris, PUF, Quadrige Dicos poche, 2004, p. 36.

${ }^{66}$ LAPlanche Jean et PONTALIS Jean-Bertrand, Fantasme originaire..., op. cit., p. 25.

${ }^{67}$ LyOTARD Jean-François, Pérégrinations, Paris, Galilée, Débats, 1990, p. 26.

${ }^{68}$ LAP LANCHE Jean et PONTALIS Jean-Bertrand, Fantasme originaire..., op. cit., p. 31.
} 
certains points à l'hypothèse d'événements réels de l'enfance, qui seraient comme des termes ultimes déguisés, pour y substituer la puissance du fantasme qui plonge dans l'instinct de mort, où tout est déjà masque et encore déguisement ${ }^{69}$.

Voilà quelques-uns des thèmes dont ont pu user les penseurs français du second XXe siècle pour « saisir » l'événement, avec tout ce que cette «saisie» conceptuelle peut comporter de paradoxe. Si la psychanalyse les a inspirés, il nous semble néanmoins qu'elle peut aussi aider à corriger cette passion du nouveau, de l'événement, du "il arrive», de l'effraction surprenante, inattendue, etc. qui accompagne souvent la pensée française et que déjà Lyotard, dans Le Différend, mettait en question chez Bataille, cette tendance, disait-il, à « choyer l'événement » (\$202). En effet, le modèle mixte de l'événement psychique qui mêle intérieur et extérieur, effraction et constitution, traumatisme et fantasme, un modèle inspiré de la psychanalyse qui n'exclut donc pas l'auto-affection par la logique propre des images inconscientes, offre encore bien des ressources aujourd'hui à une pensée plus circonspecte de l'événement.

De ce fait, gît également toute une pensée de l'image dans cette tradition psychanalytique d'inspiration freudienne. L'image n'y est pas exactement un concept mais vient désigner ce qui n'est pas événement. Toujours la dimension de l'événement, de ce qui survient sur la scène psychique, se trouve mise en tension avec la prévalence d'images mnésiques orientant les destins des représentations de pulsions. L'idée de refoulement, pivot du système psychique, principal mécanisme de défense, témoigne de cette ambivalence. Contrant la tentation d'expliquer le refoulé par le seul événement, par la rencontre d'un réel agressif, Freud introduit en 1915, dans l'article métapsychologique «Le refoulement», l'idée de refoulement originaire (Urverdrängung) qu'il distingue des post-foulements (Nachdrängen). Or le refoulé originaire, bien que Freud en dise peu de choses, désigne un refoulé sans refoulement, c'est-à-dire sans investissement de l'inconscient et sans désinvestissement du système Pcs-Cs, un refoulé qui forme le noyau de l'inconscient. Parce qu'il est le stade préliminaire ${ }^{70}$ du refoulement, le refoulé originaire constitue une couche d'images inconscientes, de " présentations pures » comme la nomme Deleuze, préalable à tout « destin » des pulsions, dans la terminologie freudienne, signe qu'il est pas de pensée de l'advenir psychique sans intervention d'images plus anciennes que tous les actes de refoulement.

\footnotetext{
${ }^{69}$ DeleuZe Gilles, Différence et répétition, Paris, PUF, Epiméthée, 1968/2003, p. 28.

${ }^{70}$ Voir FrEUD, «Inhibition, symptôme et angoisse », 1926, p. 203-286, OC XVII, op. cit., p. 212.
} 